\title{
Ocular Vascular Endothelial Heterogeneity
}

\author{
Ashima Bhattacharjee ${ }^{1}$ and Justine R. Smith ${ }^{1,2 *}$ \\ ${ }^{I}$ Casey Eye Institute/Department of Ophthalmology and ${ }^{2}$ Department of Cell \& Developmental Biology, Oregon Health \\ \& Science University, Portland, OR 97239, USA
}

\begin{abstract}
Endothelial cells form the lining of the vasculature. Despite the continuity of this layer throughout the body, endothelial cells exhibit remarkable heterogeneity in structure, molecular composition and activity: between sites; and in response to different exposures. One important consequence of endothelial diversity is the localized nature of many vascular disorders. To date a limited number of studies have attempted to define unique phenotypic features of the different intraocular endothelial subpopulations, which include the endothelial cells of vascular beds in the iris, the choroid and the retina. Differences that distinguish endothelial cells in the circulations of the choroid and the retina, in particular, are believed to be major etiological factors controlling the specific involvement of the two tissues in some of the most common blinding diseases. Age-related macular degeneration involves choroid, and diabetic retinopathy and posterior uveitis are primarily diseases of the retina. Development of effective targeted therapies for these ocular disorders will require a detailed understanding of the heterogeneity of ocular endothelia. Our review summarizes the existing literature relating to diversity of the ocular endothelial cells. We highlight structural, metabolic and functional characteristics that distinguish intraoocular endothelial subtypes from each other and from extraocular endothelial cells, and we consider the implications of these differences for the design of novel biological therapeutics for eye diseases.
\end{abstract}

Keywords: Eye, ocular, iris, choroid, retina, endothelial cell, age-related macular degeneration, diabetic retinopathy, uveitis.

\section{INTRODUCTION}

The vascular endothelium consists of the monolayer of cells and underlying basement membrane that line the blood vessels and are in direct contact with circulating blood. As recounted by Aird, in his comprehensive 2-part review of the endothelial cell $[1,2]$, Wilhelm His, a Swiss anatomist, chose the term 'endothelium' in 1865 to distinguish it from the epithelium. He used the term to denote the lining of mesothelium-lined body cavities, as well as that of blood and lymph vessels. Today, however, endothelium generally refers to the lining of the blood vessels and lymphatics. The vascular endothelium is found throughout the body and it participates in multiple processes to ensure homeostasis and/or mediate pathology. As reviewed in detail by Fajardo [3], endothelial cells (ECs): are the central cells involved in angiogenesis; have coagulant and anti-coagulant activities; facilitate immune responses and inflammatory reactions within the tissue; contribute to the regulation of vascular tone; and have certain metabolic activities. Yet, despite its continuous structure, the ECs that constitute the vascular endothelium demonstrate marked heterogeneity in structure, molecular composition and function, depending on site and over time [4]. This diversity is believed to reflect both environmental and epigenetic influences [5].

The most convincing evidence of vascular endothelial heterogeneity has come from microarray gene expression

*Address correspondence to this author at the Casey Eye Institute, Oregon Health \& Science University, 3375 SW Terwilliger Boulevard, Portland, OR 97239, USA; Tel: 503-494-5023; Fax: 503-494-6875;

E-mail: smithjus@ohsu.edu profiling studies. One comprehensive work, published in 2003 by Chi et al. [6], was an investigation of gene expression of 53 purified human EC cultures, representing 14 sites. The sites included 5 arteries, 2 veins and 7 organs or tissues (i.e. skin, lung, intestine, myometrium, lung, nasal polyps, bladder and myocardium). This work revealed heterogeneity in gene expression of: large vessel and microvascular ECs; arterial and venous ECs; and ECs from different body sites. Other studies have demonstrated that endothelial heterogeneity exists within an organ. For example, the microvasculature of the nasal mucosa consists of two EC types, termed "vascular", lying directly beneath the epithelium, and "sinusoidal", located around the nasal glands, with significant differences in respective gene expression profiles [7]. Within the heart, endocardial endothelium expresses high levels of transcripts involved in electrophysiological functions, differing from microvascular endothelium which up-regulates transcripts related to angiogenesis [8].

This review considers the topic of ocular vascular endothelial heterogeneity. Although endothelial diversity is well described for sites outside the eye, there is relatively little published about the differences between endothelial subpopulations within the eye, and the differences between ocular and extraocular ECs. After summarizing the vascular anatomy of the eye, we present clinical evidence, as well as results of in vitro and in vivo research, that confirms the existence of ocular vascular endothelial heterogeneity. We also consider the possibility of exploiting this diversity for the purposes of treatment of common causes of blindness in humans. 


\section{APPLIED VASCULAR ANATOMY OF THE EYE}

Rays of light, arriving at the anterior surface of the eye, are refracted by the transparent cornea and subsequently pass through the pupillary aperture to strike the lens. The pupil is defined by a ring-shaped muscle called the iris, which, by its constriction and dilation, controls the amount of light entering the visual organ. The cavity bordered by the cornea, iris and lens contains optically clear aqueous humor and is known as the anterior chamber. Light rays are further refracted by the transparent lens, and then pass through the vitreous humor, which fills the vitreous cavity, to arrive at the retina. Light photons are 'sensed' by the photoreceptors (i.e. rods and cones), constituting the outermost layer of the neural retina, and subsequently processed by first order neurons (i.e. bipolar cells) and second order neurons (i.e. ganglion cells). Axons of the retinal ganglion cells leave the posterior aspect of the eye as the neural component of the optic nerve. Adjacent to the photoreceptors lies an important epithelial layer known as the retinal pigment epithelium (RPE). Strauss recently reviewed the support that the RPE provides for the retinal photoreceptors [9]. Functions of the RPE include: the maintenance of local ion homeostasis, which ensures photoreceptor excitability; re-isomerization of all-trans-retinal that is produced in the photoreceptors when photons are absorbed; and promotion of photoreceptor renewal by phagocytosis of shed outer segment membranes. Beyond the RPE lies the choroid, a highly vascular, pigmented layer that separates the RPE from the external posterior coat of the eye, which is termed the sclera. The choroidal circulation is the primary source of nutrition for the outer retina, including the photoreceptors. In addition, the choroid may act as a heat sink for the highly metabolic retina and adjacent RPE.

Although the eye contains some entirely avascular tissues, including the cornea, lens and vitreous, the eye receives an ample blood supply through the ophthalmic artery, which is a branch of the internal carotid artery. As will be appreciated from any text of human anatomy, the branches of the ophthalmic artery may be divided into orbital and ocular groups, depending on the anatomical region that they supply. The ocular group, which carry arterial blood to the extraocular muscles and eye proper include: the muscular arteries, the ciliary arteries (i.e. posterior ciliary arteries and anterior ciliary arteries) and the central retinal artery. The central retinal artery passes within the optic nerve into the eye, where it traverses the lamina cribrosa of the optic disc and enters the neural retina. The 4 major branches of the central retinal artery - superior and inferior, nasal and temporal - lie on the surface of the retina and supply blood to the respective quadrants of the inner retina. Short and long posterior ciliary arteries, as described by Hayreh [10], pierce the sclera to reach the choroid; choroidal end-arteries give rise to separate lobules of the extensive choriocapillaris that constitutes the bulk of the choroid and supplies the inner retina. Posterior ciliary arteries are also the source of the arterial circulation of the anterior part of the optic nerve, and they contribute to the blood supply of the iris and ciliary body. Anterior ciliary arteries enter the eye close to the sclerocorneal junction and are the primary source of arterial supply to the anterior seg- ment. The major intraocular arterial vessels of the anterior eye are the peripheral 'greater arterial circle of the iris', located at the root of the iris, and the 'lesser arterial circle of the iris', which lies near the pupil margin; these circles are connected by radial vessels. Although the arterial vessels destined for the choroid and the ciliary body course through the sclera, this layer of the eye does not receive an extensive arterial supply. The branches of veins draining the 4 quadrants of the retina join at the optic disc to form the central retinal vein, which feeds into the cavernous sinus either directly or via the superior ophthalmic vein. Veins from the choroid, ciliary body and iris converge to form 4 to 6 principal vortex veins, which exit the sclera near the equator of the eye to drain into the superior or inferior ophthalmic veins. Venous blood in the iris and ciliary body may also be carried from these tissues in small anterior and posterior ciliary veins, which ultimately drain into the superior ophthalmic vein.

Diversity of the different ocular endothelial subpopulations is apparent at an ultrastructural level. This specialization is tied, in part, to the blood-ocular barriers, which like the blood-brain barrier, exist to regulate the transfer of molecules and cells between the blood stream and the intraocular tissues. The classic review published in 1979 in Survey of Ophthalmology by Cunha-Vaz [11] describes these barriers much as we understand them today, i.e. the blood-aqueous barrier and the blood-retinal barrier. Both barriers include an epithelial and an endothelial component. Iris ECs are a critical component of the blood-aqueous barrier, which is located in the anterior eye. The non-pigmented ciliary epithelium and the contiguous posterior iris epithelium form the second component of this barrier. Saari studied the ultrastructure of iris ECs from several mammals and showed that in the cat, rat, rabbit and guinea pig, the endothelium of iris microvessels was devoid of fenestrations; he cited reports from the 1960s that described a similar observation for human iris ECs [12]. However, iris vessels are more permeable than retinal vessels [11]. Saari described the presence of relatively wide intercellular space and macula occludens, which he concluded might allow passage of water and low molecular weight solutes [12]. Although ciliary arteriolar ECs are nonfenestrated, the ECs of the ciliary capillaries and venules are fenestrated, and they present discontinuous junctional complexes that are not sealed at the interendothelial clefts [13]. The blood-retinal barrier is composed of the retinal endothelium and the RPE. Retinal ECs are non-fenestrated and are connected via zonula occludens or "non-leaky tight junctions"; on the other hand, the choroidal ECs contain fenestrations, which are particularly numerous on their retinal aspect $[11,14]$. Interestingly, the retinal ECs exhibit morphological heterogeneity depending on their location in the vascular bed, as demonstrated in studies carried out in porcine eyes [15]. The ECs become smaller and more rounded from large arteriole to small arteriole, to capillary, to venule. The nucleus is positioned quite centrally in the largest arterioles, but is displaced away from the center, following the direction of blood flow, in smaller arterioles; in venules the nucleus is found near the center of the cell. Yu et al. [15] who made these observations, considered them supportive of a role for retinal ECs in the regulation of retinal blood flow. 


\section{CLINICAL OBSERVATIONS: DIFFERENT OCULAR DISEASES INVOLVE DIFFERENT VASCULAR BEDS}

The existence of ocular vascular endothelial diversity is reflected in the manifestations of various vascular, inflammatory and malignant diseases that affect the eye. Different ocular diseases involve different vascular beds, and the different endothelial subpopulations play a critical role in the localization of specific diseases. Ocular vascular diseases are leading causes of irreversible vision loss in both industrialized and developing nations [16]. In Western countries, agerelated macular degeneration (AMD) is the leading cause of blindness in persons aged 50 years and older [17]. Advanced disease presents as 2 forms: 'non-neovascular AMD', characterized by deposits known as drusen and disruption of the epithelium; and 'neovascular AMD', in which new vessels proliferate in the subretinal space, causing vision loss in a majority of patients [18]. These new vessels originate when choroidal ECs proliferate and migrate in response to endogenous angiogenic and inflammatory mediators [19]. Retinal endothelium is not involved. In contrast, when diabetes mellitus affects the eye, the disease is localized to the retinal vasculature, and retinal, but not choroidal, ECs are critically involved. Approximately two-thirds of patients with type 1 diabetes and one-third of patients with type 2 diabetes have retinopathy [20]. The loss of retinal ECs and associated pericytes is accompanied by formation of microaneurysms and increased retinal vascular permeability [20,21]. Such changes result in the hemorrhages and leakage of fluid, protein and lipid into retina that characterize 'background retinopathy'. The disease progresses to 'proliferative retinopathy' when retinal ECs form new blood vessels in response to angiogenic factors released in the context of hypoxia. Retinopathy of prematurity is a second disease that is characterized by neovascularization in the retina, although it is manifest in premature infants as a result of exposure to high oxygen tensions outside the uterus [22]. Hypertension and atherosclerosis also preferentially affect the retinal vessels and their endothelial lining.

Inflammatory conditions and a number of endogenous infectious and malignant diseases characteristically affect specific ocular tissues. This differential disease localization implies involvement of a specific vascular bed and its respective endothelial subpopulation in any given disease, since homing is critical for these blood-borne diseases. Uveitis refers to inflammatory disease affecting the intraocular tissues. Although the condition is relatively uncommon [23], vision loss affects approximately $35 \%$ of affected individuals [24]. Uveitis is defined anatomically to designate that the primary site of the inflammation; the iris and/or ciliary body are inflamed in anterior uveitis, while posterior uveitis is an inflammation of the retina and/or choroid [25]. The inflammation may be immune-mediated and associated with systemic diseases that include the sero-negative spondyloarthropathies and juvenile idiopathic arthritis, in anterior uveitis, and sarcoidosis and Behcet's disease, in posterior uveitis [26]. The commonest blood-borne infectious uveitis is ocular toxoplasmosis or infection with the parasite, Toxoplasma gondii [26]. In this disease, inflammation is localized to the retina. Interestingly, when uveitis involves the retina, which contains a circulation that can be visualized clinically, infiltrates are frequently visible along the retinal vessels. Preferential disease localization is also observed in several ocular malignancies. Intraocular lymphoma is a rare tumor that is based in the retina [27]. Furthermore, although it may involve brain, it does not generally metastasize outside the central nervous system. The most plausible explanation of this distribution is a preferential homing of malignant $\mathrm{B}$ cells to vessels in the brain and retina [28]. In contrast, the vast majority of metastatic tumors, including secondary deposits from breast and lung carcinomas, are found in the choroid [29].

\section{OCULAR VASCULAR ENDOTHELIAL HETERO- GENEITY: EVIDENCE FROM IN VITRO STUDIES}

A limited number of profiling studies have evaluated the molecular phenotype of different ocular EC populations. Silverman et al. [30] used two cDNA arrays to compare primary human iris and retinal EC with regard to expression of one set of 588 genes relating to various cell functions and a second set of 406 genes encoding proteins with hematologic/immunologic activities. Using these small arrays, the profiles of the two cell populations differed in the absence of stimulation, but these differences were not completely described, as the study focused on the effects of inflammatory stimulation, as described later in this section. In 2007, we used oligonucleotide arrays designed to detect 8746 wellcharacterized transcripts to compare expression profiles of donor-matched human retinal and choroidal ECs from 6 individuals [31]. Approximately $9 \%$ of genes were differentially expressed by unstimulated ECs, according to the significance analysis of microarrays (SAM) with false discovery rate equal to $5 \%$. Differential expression of genes by retinal and choroidal EC has also been reported for bovine eyes studied by differential display RT-PCR, although the majority of transcripts were not identified [32]. In our study [31], gene ontology identified a relatively greater percentage of transcripts associated with the immune response in the group of genes expressed at higher levels in retinal EC. Some of these transcripts encoded cell adhesion molecules (i.e. intercellular adhesion adhesion molecule [ICAM]-1, vascular cell adhesion molecule [VCAM]-1, E-selectin) and chemokines (i.e. CCL2, CXCL6, CXCL8, CX3CL1). This finding is consistent with the predominant involvement of the retinal vasculature in the development of posterior uveitis. One important observation in this study was that gene expression varied markedly between donors, emphasizing the importance of using donor-matched cultures in profiling studies involving ECs from any site in the body.

Proteomics profiling confirms that diversity in ocular endothelial molecular phenotype is manifested at the level of protein expression. In 2007, we undertook a study to characterize the proteome of human retinal and choroidal ECs by comparing protein extracts from ECs isolated from eyes of five cadavers, using 2-dimensional difference gel electrophoresis was combined with tandem mass spectrometry [33]. In this study, 123 protein spots on the gels were suitable for analysis, and approximately $25 \%$ of these spots were differentially expressed by SAM. Given that angiogenesis is the central pathogenic process in proliferative diabetic retinopathy, affecting the retina, and AMD, affecting the choroid, it was noteworthy that a number of proteins with the potential to influence new vessel growth were differentially expressed. 
These included calreticulin and cathepsin $\mathrm{B}$, which were significantly more abundant in retinal EC, and glutathione peroxidase 1, ubiquitin carboxyl-terminal hydrolase isozyme $\mathrm{L} 1$ and superoxide dismutase (Cu-Zn) (SOD1), which were significantly more abundant in choroidal EC. In an earlier study using bovine eyes, Brylla et al. [32] showed that transcript encoding vascular endothelial growth factor (VEGF) receptor, VEGF-R1, was relatively high in retinal EC, while expression of VEGFR-2 transcript was relatively low, in comparison to levels in choroidal EC. Gene expression for the Tie-2 ligand, angiopoetin (Ang)-2, was also higher in retinal EC, although Ang-1 was not differentially expressed. These independent observations are consistent with the concept that angiogenesis is differentially regulated in the retinal and choroidal vascular beds, and that retinal and choroidal vascular diseases are controlled by different molecular mediators, as previously suggested by Campochiaro in his review of ocular angiogenesis [34].

Differences in the transcriptomes and proteomes of ocular ECs may be related, at least in part, to differences in the activity of specific transcription factors. To study this issue in relation to retinal and choroidal ECs, we conducted an in silico analysis of data generated in the microarray study that is described in a preceding paragraph [35]. This investigation led to the identification of certain transcription factor recognition motifs that were significantly more abundant in genes showing differential expression in retinal vs. choroidal ECs. The 3 motifs - corresponding to transcription factor E2F, Yin Yang1 and zinc finger 5 - were more abundant in the promoters of the genes that were highly expressed in the choroidal ECs. In the paper we speculated that the named transcription factors might participate in some key events in the development of AMD, i.e. mitochondrial dysfunction and overproduction of vascular endothelial growth factor (VEGF) and EC proliferation. Similarly, the transcription factors corresponding to motifs present in high abundance in the promoters of genes that are highly expressed in retinal ECs - corresponding to glucocorticoid receptor, high mobility group AT-hook 1, heat shock transcription factor 1, p53 and vitamin D receptor - impact cell proliferation, apoptosis, aging and the stress response and consequently might influence neovascularization in proliferative diabetic retinopathy. Differentially expressed retinal and choroidal EC genes also could be distinguished on the basis of distinct cis-regulatory modules. These observations add to understanding of the molecular basis for the selective involvement of the retinal and choroidal vascular beds in diabetic retinopathy and AMD, respectively.

Diversity of the ocular EC molecular phenotype is also apparent in the responses of ocular ECs to various molecular and biological stimuli. Retinal ECs appear to be particularly susceptible to oxidative stress, which may explain their involvement in the microvasculopathy of diabetes mellitus [36]. In comparison to bovine brain-derived ECs, retinal ECs demonstrate a relatively small increase in the production of the antioxidant enzyme, glutathione peroxidase, after exposure to elevated glucose concentrations; retinal ECs incubated in glucose at a physiological concentration make relatively low levels of this enzyme and a second antioxidant enzyme, superoxide dismutase, but generate relatively high levels of superoxide. Moreover, retinal ECs, unlike brainderived ECs, do not increase their production of the junctional protein, ZO-1, after exposure to high levels of glucose, which equates with increased vascular permeability in this setting. Separate research has shown that survival of cultured bovine choroidal ECs is compromised when oxidative stress is induced by exposure to tert-butyl-hydroperoxide, and that the cells may be rescued by soluble mediators secreted by RPE, with implications for the pathogenesis of AMD [37]. Comparative studies of retinal and choroidal EC responses to oxidative stress have not been published, however.

In the microarray study by Silverman et al. [30] stimulation of iris and retinal ECs with the inflammatory cytokine, tumor necrosis factor (TNF)- $\alpha$, resulted in significantly higher levels of gene expression for cell adhesion molecules, E-selectin and VCAM-1, and the chemokine, CCL2, in donor-matched human retinal ECs $v s$. iris ECs. The differential expression of E-selectin was confirmed at the protein level, and E-selectin blocking antibodies prevented binding of U937 monocytes to human retinal ECs. Our microarray investigation identified other differences in the expression by human retinal and choroidal endothelial of genes encoding proteins involved in the immune response after exposure to inflammatory stimuli [31] One notable example was the stimulation of ECs with $T$. gondii tachyzoites, which induced up-regulation of ICAM-1 gene expression by the retinal, but not the choroidal, endothelial subpopulations. Since ICAM-1 may act as a receptor for tachyzoites or parasitized leukocytes crossing the blood-retinal barrier, this finding may be directly relevant to the localization of ocular toxoplasmosis to the retina.

To date, research that translates the molecular distinctions reported between different ocular ECs and between ocular and extraocular EC subpopulations into functional differences is scant. At least 1 study supports the concept of differential angiogenic mechanisms in retinal vs. choroidal EC. In contrast to retinal vessels, choroidal blood vessels receive a rich sympathetic innervation, and loss of this innervation leads to new vessel growth; these observations led Steinle and Granger to suggest the hypothesis that nerve growth factor (NGF) - a factor with angiogenic properties that is associated with sympathetic neurons - differentially regulated angiogenesis in the retina and choroid [38]. They were able to demonstrate significant increase in the migration and proliferation of cultured human choroidal, but not retinal, ECs, in response to exogenous NGF, an effect that appeared to be mediated by phosphorylation of its receptor, TrkA, as well as signaling proteins Src, Akt and MAPK. Work from our laboratory has shown that cultured human retinal EC are preferentially susceptible to infection with $T$. gondii tachyzoites in comparison to human ECs from extraocular sites, including aortic ECs, human umbilical venous ECs and dermal ECs [39]. The human retinal endothelium is capable of binding tachyzoites in a modified Woodruff-Stamper assay [40], and the relative susceptibility of cultured human retinal ECs to infection reflects an enhanced parasite-host EC binding and penetration, at least in comparison to dermal ECs [41]. It is tempting to speculate a role for ICAM-1 in this process, as discussed above. 


\section{OCULAR VASCULAR ENDOTHELIAL HETERO- GENEITY: EVIDENCE FROM IN VIVO STUDIES}

Investigations utilizing animal models of uveitis have provided strong in vivo evidence of vascular endothelial diversity within the eye. Multiple forms of experimental uveitis have been described. When rodents are injected systemically or locally with lipopolysaccharide, a transient inflammatory infiltrate with high numbers of neutrophils and macrophages is observed in the anterior segment of the eye; this response is termed endotoxin-induced uveitis (EIU) [42, 43]. The leukocytes that mediate an inflammatory reaction must cross the endothelium of the vasculature in order to gain access to the tissue. This process involves several steps including rolling, firm adhesion and transmigration [44]. Intravital microscopic studies of 3 vascular beds in the eyes of $\mathrm{BALB} / \mathrm{c}$ mice - peripheral corneal (or limbal), iris and anterior choroidal - have demonstrated clear differences in the kinetics of rolling and firm adhesion during the leukocyte-EC interaction in the different vascular beds in EIU [45]. We suggest that these observations may be explained, at least in part, by different patterns of expression of cell adhesion molecules on ECs in the different vascular beds. In humans, many cases of uveitis are believed to be mediated by $\mathrm{CD} 4+\mathrm{T}$ cells; $\mathrm{T}$ cell-mediated models of anterior and posterior uveitis have been described. One well established model of posterior uveitis, known as experimental autoimmune uveoretinitis (EAU), is induced in rats or mice by systemic immunization with one of several retinal antigens [46]. Elegant work conducted 10 years ago by Parnaby-Price et al. [47], involving intravital ophthalmoscopy and confocal microscopic study of retinal whole-mounts, clearly demonstrated the existence of endothelial diversity in the posterior eye; leukocytes trafficked into the eye via the retinal, not the choroidal, vasculature in this model. More recent work applying similar methodology to the same model has localized breakdown of the blood-retinal barrier to the retinal post-capillary venules [48]. This observation indicates the existence of additional endothelial diversity - arterial $v s$. venous - within the retinal circulation. Retinal venular endothelium expresses increased levels of P-selectin, E-selectin, ICAM-1 and perhaps CD44 at the onset of EAU, implicating these cell adhesion molecules in the extravasation of the Tcells $[48,49]$.

Experimental models of ocular diseases that involve neovascularization are also illustrative of ocular vascular endothelial diversity. As they age, mice deficient in the chemokine, CCL2 - also known as monocyte chemoattractant protein-1 - or its receptor, CCR2, develop ocular lesions that clinically, histologically and angiographically resemble the lesions of human AMD, including the spontaneous development of choroidal neovascularization in approximately one-quarter of the animals [50]. When the CCL2 gene knock-out mice are additionally deficient in the receptor for another chemokine, $\mathrm{CX}_{3} \mathrm{CL} 1$ - also known as fractalkine - lesions develop at an earlier age [51]. $\mathrm{CX}_{3} \mathrm{CR} 1$ gene deficient mice also develop AMD-like lesions, but choroidal neovascularization appears to require choroidal laser photocoagulation injury [52]. Choroidal laser photocoagulation in various animal species is an established method for induction of experimental choroidal neovascularization for the study of the mechanisms operating in AMD. These independent observations implicate chemokines in the development of AMD. In work from other laboratories, it is clear that SOD1 provides protection from choroidal neovascularization. As reported in a preceding paragraph, we found this anti-oxidant to be highly expressed by unstimulated human choroidal ECs. Genetic deficiency of SOD1 causes aged mice to develop an AMD-like picture, including spontaneous new vessel formation from the choroid in approximately $10 \%$ of animals [53]. In a recently described, novel mouse model of non-neovascular AMD, mice deficient in gene encoding CD36 show involution of choroidal vessels; CD36 is expressed on RPE where it participates in phagocytosis [54]. Importantly, in all the mouse models described in this paragraph, the vascular abnormalities appear to be limited to the choroidal vasculature.

Various animal models have been reported for studying the pathogenesis of common retinal vascular diseases, i.e. diabetic retinopathy, retinal vein occlusion and retinopathy of prematurity. In the models described below, the choroidal vasculature is not involved. Rodents and other mammals injected systemically with streptozotocin develop a diabetic syndrome that is characterized by the vascular changes also observed in patients with background diabetic retinopathy [55]. Intravitreal injection of adenoviral vector encoding VEGF may induce iris and inner retinal neovascularization in the eyes of rhesus monkeys [56]. Very recently, Zhang et al. [57] presented a rat model of retinal vein - central or branch - occlusion, which was adapted from other published animal models. They performed diode green laser photocoagulation to occlude retinal veins that were visualized at the slit lamp following intravenous injection of Rose Bengal. The murine model of retinopathy of prematurity is known as oxygen-induced retinopathy (OIR) [58]. At birth, the retina of the mouse is incompletely vascularized. In this model, neonatal mice are exposed to hyperoxia for approximately 5 days and then maintained in room air. Such treatment induces the formation of neovascular tufts at the boundary of vascular and avascular retina. Using a similar model in the rat, it has been shown that the Kreb's cycle intermediate, succinate, regulates this neovascularization; succinate binds its cognate receptor, GPR91, on retinal ganglion cells and stimulates these neurons to release angiogenic factors, including VEGF, Ang-1 and Ang-2 [59].

\section{EXPLOITING OCULAR VASCULAR ENDOTHELIAL DIVERSITY TO TREAT DISEASES OF THE EYE}

The existence of molecular, structural and functional differences between ECs of the eye provides an opportunity to target drugs at specific EC subpopulations. In theory, this should allow the treatment of a disease that involves 1 ocular vascular bed, without impacting an adjacent healthy vasculature. Recently treatments that target ocular ECs have become a reality in the clinic, facilitated by local options for drug delivery. Although it is not specific for one vascular bed, VEGF is the target of the first biologic agents for ocular neovascular disease, after extensive literature demonstrated its role in diseases including neovascular AMD and proliferative diabetic retinopathy. This topic is reviewed in detail by Zhang and Ma [60]. The US Federal Drug Administration has approved the use of pegaptanib - an aptamer that targets extracellular VEGF165 - and ranibizumab - a humanized 
monoclonal Fab that binds all isoforms of VEGF - by intravitreal injection for the treatment of wet AMD, following successful Phase III trials [61]. Although less rigorously studied to date, published reports suggest that VEGF blockade also has therapeutic benefit for proliferative diabetic retinopathy [62]. Indeed, vitreo-retinal surgeons are injecting bevacizumab - an anti-VEGF monoclonal antibody related to ranibizumab - intravitreally as part of the preoperative preparation for patients who require vitrectomy for their disease [63]. Targeting of the ocular vasculature is also being considered for inflammatory eye disease. A Phase II study that is presently enrolling at the National Eye Institute will evaluate the therapeutic effect of efalizumab in patients with macular edema complicating posterior or intermediate uveitis (clinicaltrials.gov: identifier $=$ NCT00280826); efalizumab is a humanized monoclonal antibody that, by targeting CD11a, prevents interaction between lymphocyte function antigen (LFA)-1 and ICAM-1 and thereby blocks leukocyticendothelial adhesion.

Endothelial subtype-specific therapies are yet to be applied clinically to eye diseases, but there are many pieces of evidence that support the concept of differential effects of potential therapeutic agents on extraocular vs. intraocular endothelium. For example, cathepsin B has angiosuppressive effects in capillary tube formation assays using bovine retinal ECs and in retinal vessel outgrowth assays using murine retinas; these effects contrast starkly with its established role as a promoter of angiogenesis in tumors [64]. Examples from the literature indicate that ocular vascular endothelial diversity is also likely to impact treatment of eye diseases that involve the vasculature. As highlighted by Campochiaro in his review of ocular neovascularization [34], alpha-interferon- $2 b$ is an effective treatment for experimental ischemic iris neovascularisation in cynomolgus monkeys [65]. In contrast, interferon alfa-2a, administered to patients with AMDrelated choroidal neovascularization, not only did not improve the disease, but at some doses was found to worsen it, in comparison to disease in the patients who were treated with placebo [66]. The therapeutic effect of an exogenous agent may also depend on the specific conditions under which it is administered. Angiopoietin-2, when overexpressed in neonatal mice exposed to hypoxia, leads to the formation of retinal new vessels. The same agent, when applied after return to normoxia, causes regression of the new vessels [67]. As discussed above, we identified high expression of ICAM-1 by retinal ECs. Consistent with this observation, while monoclonal antibodies against ICAM-1 inhibit inflammatory infiltration of the retina in EAU [68], they do not inhibit experimental melanin-induced uveitis, a T cell-mediated model of anterior uveitis in which leukocytes traffic though the iris endothelium [69].

\section{CONCLUSIONS}

Definition of the phenotype of the vascular ECs of the eye is an area of research that has lagged behind the work conducted on these cells in other organ systems. Yet now, accumulating evidence indicates that vascular endothelial heterogeneity is manifest in both the molecular profile and the function of ECs within the human eye. The EC is a central player in the pathogenesis of leading causes of blindness, including AMD, diabetic retinopathy and posterior uveitis.
Several lines of investigation suggest that the documented endothelial diversity is likely to impact the basic processes responsible for and to have implications for therapeutic approaches to these diseases.

Studies of ocular endothelial diversity have been facilitated by methods to isolate ECs from different circulations of the eye in a single donor [30, 31, 33, 35]. However, both genetic factors and environmental conditions determine the phenotype of any given EC. While microarray surveys of cultured ECs provide strong evidence that some aspects of cell phenotype are not dependent on environmental cues [68,31 ], studies comparing freshly isolated and cultured cells clearly indicate that the microenvironment plays a role in maintaining other phenotypic features. Durr et al. [70] used proteomics to quantify the difference in protein expression by freshly isolated $v s$. cultured rat lung ECs; of 450 proteins identified in a rigorous analysis, $41 \%$ of proteins that were detected in vivo were not detected in vitro. Progress in the field will therefore require approaches that complement studies with cultured human ocular ECs. Animal models of ocular disease have a role in this investigation, but speciesrelated differences in endothelial phenotype must be recognized. For example, prostate-specific membrane antigen is expressed on the endothelium of human tumor vasculature but not in comparable vessels in the mouse [71]. Techniques such as laser capture microdissection of endothelial populations in fresh healthy or diseased tissue [72], combined with molecular amplification methods, and in vivo phage display $[73,74]$ provide exciting future opportunities to study the diversity of human ocular ECs in their native environment.

\section{ACKNOWLEDGEMENTS}

The authors thank James T. Rosenbaum, MD, for his insightful critique of their manuscript.

This work was supported in part by Research to Prevent Blindness and the Schnitzer-Novack Foundation.

\section{REFERENCES}

[1] Aird WC. Phenotypic heterogeneity of the endothelium: I. Structure, function, and mechanisms. Circ Res 2007; 100: 158-73.

[2] Aird WC. Phenotypic heterogeneity of the endothelium: II. Representative vascular beds. Circ Res 2007; 100: 174-90.

[3] Fajardo LF. The complexity of endothelial cells: a review. Am J Clin Pathol 1989; 92: 241-50.

[4] Aird WC. Spatial and temporal dynamics of the endothelium. J Thromb Haemost 2005; 3: 1392-406.

[5] Aird WC. Mechanisms of endothelial cell heterogeneity in health and disease. Circ Res 2006; 98: 159-62.

[6] Chi JT, Chang HY, Haraldsen G, et al. Endothelial cell diversity revealed by global expression profiling. Proc Natl Acad Sci USA 2003; 100: 10623-8.

[7] Holmen C, Stjarne P, Sumitran-Holgersson S. Heterogeneity of human nasal vascular and sinusoidal endothelial cells from the inferior turbinate. Am J Respir Cell Mol Biol 2005; 32: 18-27.

[8] Hendrickx J, Doggen K, Weinberg EO, Van Tongelen P, Fransen P, De Keulenaer GW. Molecular diversity of cardiac endothelial cells in vitro and in vivo. Physiol Genomics 2004; 19: 198-206.

[9] Strauss O. The retinal pigment epithelium in visual function. Physiol Rev 2005; 85: 845-81.

[10] Hayreh SS. Segmental nature of the choroidal vasculature. Br J Ophthalmol 1975; 59: 631-48.

[11] Cunha-Vaz J. The blood-ocular barriers. Surv Ophthalmol 1979; 23: 279-96. 
[12] Saari M. Ultrastructure of the microvessels of the iris in mammals with special reference to their permeability. Albrecht Von Graefes Arch Klin Exp Ophthalmol 1975; 194: 87-93.

[13] Hirsch M, Renard G, Faure JP, Pouliquen Y. Endothelial cell junctions in the ciliary body microvasculature. A freeze-fracture study in the rabbit. Albrecht Von Graefes Arch Klin Exp Ophthalmol 1978; 208: 69-76.

[14] Hogan MJ, Alvarado JA, Weddell JE. Histology of the Human Eye. An Atlas and Textbook. Philadelphia: WB Saunders Co 1971.

[15] Yu PK, Yu D, Alder VA, Seydel U, Su E, Cringle SJ. Heterogeneous endothelial cell structure along the porcine retinal microvasculature. Exp Eye Res 1997; 65: 379-89.

[16] Congdon NG, Friedman DS, Lietman T. Important causes of visual impairment in the world today. JAMA 2003; 290: 2057-60.

[17] Pascolini D, Mariotti SP, Pokharel GP, et al. 2002 global update of available data on visual impairment: a compilation of populationbased prevalence studies. Ophthalmic Epidemiol 2004; 11: 67-115.

[18] Jager RD, Mieler WF, Miller JW. Age-related macular degeneration. N Engl J Med 2008; 358: 2606-17.

[19] Grisanti S, Tatar O. The role of vascular endothelial growth factor and other endogenous interplayers in age-related macular degeneration. Prog Retin Eye Res 2008; 27: 372-90.

[20] Frank RN. Diabetic retinopathy. N Engl J Med 2004; 350: 48-58.

[21] Davidson JA, Ciulla TA, McGill JB, Kles KA, Anderson PW. How the diabetic eye loses vision. Endocrine 2007; 32: 107-16.

[22] Rubaltelli DM, Hirose T. Retinopathy of prematurity update. Int Ophthalmol Clin 2008; 48: 225-35.

[23] Gritz DC, Wong IG. Incidence and prevalence of uveitis in Northern California; The Northern California Epidemiology of Uveitis Study. Ophthalmology 2004; 111: 491-500; discussion 500.

[24] Rothova A, Suttorp-van Schulten MS, Frits Treffers W, Kijlstra A. Causes and frequency of blindness in patients with intraocular inflammatory disease. Br J Ophthalmol 1996; 80: 332-6.

[25] Jabs DA, Nussenblatt RB, Rosenbaum JT. Standardization of uveitis nomenclature for reporting clinical data. Results of the First International Workshop. Am J Ophthalmol 2005; 140: 509-16.

[26] Rodriguez A, Calonge M, Pedroza-Seres M, et al. Referral patterns of uveitis in a tertiary eye care center. Arch Ophthalmol 1996; 114: 593-9.

[27] Gunduz K, Pulido JS, McCannel CA, O'Neill BP. Ocular manifestations and treatment of central nervous system lymphomas. Neurosurg Focus 2006; 21: E9.

[28] Hochberg FH, Miller DC. Primary central nervous system lymphoma. J Neurosurg 1988; 68: 835-53.

[29] Ou JI, Wheeler SM, O'Brien JM. Posterior pole tumor update. Ophthalmol Clin N Am 2002; 15: 489-501.

[30] Silverman MD, Babra B, Pan Y, Planck SR, Rosenbaum JT. Differential E-selectin expression by iris vs. retina microvascular endothelial cells cultured from the same individuals. Microvasc Res 2005; 70: 32-42.

[31] Smith JR, Choi D, Chipps TJ, et al. Unique gene expression profiles of donor-matched human retinal and choroidal vascular endothelial cells. Invest Ophthalmol Vis Sci 2007; 48: 2676-84.

[32] Brylla E, Tscheudschilsuren G, Santos AN, Nieber K, SpanelBorowski K, Aust G. Differences between retinal and choroidal microvascular endothelial cells (MVECs) under normal and hypoxic conditions. Exp Eye Res 2003; 77: 527-35.

[33] Zamora DO, Riviere M, Choi D, et al. Proteomic profiling of human retinal and choroidal endothelial cells reveals molecular heterogeneity related to tissue of origin. Mol Vis 2007; 13: 2058-65.

[34] Campochiaro PA. Ocular vs. extraocular neovascularization: mirror images or vague resemblances. Invest Ophthalmol Vis Sci 2006; 47: 462-74.

[35] Choi D, Appukuttan B, Binek SJ, et al. Prediction of cis-regulatory elements controlling genes differentially expressed by retinal and choroidal vascular endothelial cells. J Ocul Biol Dis Inform 2008; $1: 37-45$.

[36] Grammas P, Riden M. Retinal endothelial cells are more susceptible to oxidative stress and increased permeability than brainderived endothelial cells. Microvasc Res 2003; 65: 18-23.

[37] Eichler W, Reiche A, Yafai Y, Lange J, Wiedemann P. Growthrelated effects of oxidant-induced stress on cultured RPE and choroidal endothelial cells. Exp Eye Res 2008; 87: 342-8.

[38] Steinle JJ, Granger HJ. Nerve growth factor regulates human choroidal, but not retinal, endothelial cell migration and proliferation. Auton Neurosci 2003; 108: 57-62.
[39] Smith JR, Franc DT, Carter NS, Zamora D, Planck SR, Rosenbaum JT. Susceptibility of retinal vascular endothelium to infection with Toxoplasma gondii tachyzoites. Invest Ophthalmol Vis Sci 2004; 45: 1157-61.

[40] Chipps TJ, Streeter PR, Franc DT, et al. Modification of the Woodruff-Stamper assay demonstrates binding of Toxoplasma gondii tachyzoites to retinal vascular endothelium. J Immunol Methods 2006; 312: 209-13

[41] Zamora DO, Rosenbaum JT, Smith JR. Invasion of human retinal vascular endothelial cells by Toxoplasma gondii tachyzoites. Br J Ophthalmol 2008; 92: 852-5.

[42] Rosenbaum JT, McDevitt HO, Guss RB, Egbert PR. Endotoxininduced uveitis in rats as a model for human disease. Nature 1980 286: 611-3.

[43] Rosenbaum JT, Rosenzweig HL, Smith JR, Martin TM, Planck SR. Uveitis secondary to bacterial products. Ophthalmic Res 2008; 40: 165-8.

[44] Crane IJ, Liversidge J. Mechanisms of leukocyte migration across the blood-retina barrier. Semin Immunopathol 2008; 30: 165-77.

[45] Rosenbaum JT, Brischetto M, Crespo S, et al. Simultaneous in vivo imaging of leukocyte migration: heterogeneity among iris, limbus, and choroid vessels. Curr Eye Res 2002; 24: 214-8.

[46] Nussenblatt RB. Proctor Lecture. Experimental autoimmune uveitis: mechanisms of disease and clinical therapeutic indications. Invest Ophthalmol Vis Sci 1991; 32: 3131-41.

[47] Parnaby-Price A, Stanford MR, Biggerstaff J, et al. Leukocyte trafficking in experimental autoimmune uveitis in vivo. J Leukoc Biol 1998; 64: 434-40.

[48] Xu H, Forrester JV, Liversidge J, Crane IJ. Leukocyte trafficking in experimental autoimmune uveitis: breakdown of blood-retinal barrier and upregulation of cellular adhesion molecules. Invest Ophthalmol Vis Sci 2003; 44: 226-34.

[49] Xu H, Manivannan A, Jiang HR, et al. Recruitment of IFN-gammaproducing (Th1-like) cells into the inflamed retina in vivo is preferentially regulated by $\mathrm{P}$-selectin glycoprotein ligand $1: \mathrm{P} / \mathrm{E}-$ selectin interactions. J Immunol 2004; 172: 3215-24.

[50] Ambati J, Anand A, Fernandez S, et al. An animal model of agerelated macular degeneration in senescent Ccl-2- or Ccr-2-deficient mice. Nat Med 2003; 9: 1390-7.

[51] Tuo J, Bojanowski CM, Zhou M, et al. Murine $\mathrm{ccl} 2 / \mathrm{cx} 3 \mathrm{cr} 1$ deficiency results in retinal lesions mimicking human age-related macular degeneration. Invest Ophthalmol Vis Sci 2007; 48: 3827 36.

[52] Combadiere C, Feumi C, Raoul W, et al. CX3CR1-dependent subretinal microglia cell accumulation is associated with cardinal features of age-related macular degeneration. J Clin Invest 2007; 117: 2920-8

[53] Imamura Y, Noda S, Hashizume K, et al. Drusen, choroidal neovascularization, and retinal pigment epithelium dysfunction in SOD1-deficient mice: a model of age-related macular degeneration. Proc Natl Acad Sci USA 2006; 103: 11282-7.

[54] Houssier M, Raoul W, Lavalette S, et al. CD36 deficiency leads to choroidal involution via COX2 down-regulation in rodents. PLoS Med 2008; 5: e39.

[55] Feit-Leichman RA, Kinouchi R, Takeda M, et al. Vascular damage in a mouse model of diabetic retinopathy: relation to neuronal and glial changes. Invest Ophthalmol Vis Sci 2005; 46: 4281-7.

[56] Lebherz C, Maguire AM, Auricchio A, et al. Nonhuman primate models for diabetic ocular neovascularization using AAV2mediated overexpression of vascular endothelial growth factor. Diabetes 2005; 54: 1141-9.

[57] Zhang Y, Fortune B, Atchaneeyasakul LO, et al. Natural history and histology in a rat model of laser-induced photothrombotic retinal vein occlusion. Curr Eye Res 2008; 33: 365-76.

[58] Smith LE, Wesolowski E, McLellan A, et al. Oxygen-induced retinopathy in the mouse. Invest Ophthalmol Vis Sci 1994; 35: 101-11.

[59] Sapieha P, Sirinyan M, Hamel D, et al. The succinate receptor GPR91 in neurons has a major role in retinal angiogenesis. Nat Med 2008; 14: 1067-76.

[60] Zhang SX, Ma JX. Ocular neovascularization: Implication of endogenous angiogenic inhibitors and potential therapy. Prog Retin Eye Res 2007; 26: 1-37.

[61] Kaiser PK. Antivascular endothelial growth fctor agents and their development: therapeutic implications in ocular disease. Am J Ophthalmol 2006; 142: 660-68. 
[62] Adamis AP, Altaweel M, Bressler NM, et al. Changes in retinal neovascularization after pegaptanib (Macugen) therapy in diabetic individuals. Ophthalmology 2006; 113: 23-8.

[63] Simo R, Hernandez C. Intravitreous anti-VEGF for diabetic retinopathy: hopes and fears for a new therapeutic strategy. Diabetologia 2008; 51: 1574-80.

[64] Im E, Venkatakrishnan A, Kazlauskas A. Cathepsin B regulates the intrinsic angiogenic threshold of endothelial cells. Mol Biol Cell 2005; 16: 3488-500

[65] Miller JW, Stinson WG, Folkman J. Regression of experimental iris neovascularization with systemic alpha-interferon. Ophthalmology 1993; 100: 9-14.

[66] Interferon alfa-2a is ineffective for patients with choroidal neovascularization secondary to age-related macular degeneration. Results of a prospective randomized placebo-controlled clinical trial. Pharmacological Therapy for Macular Degeneration Study Group. Arch Ophthalmol 1997; 115: 865-72.

[67] Oshima Y, Oshima S, Nambu H, et al. Different effects of angiopoietin-2 in different vascular beds: new vessels are most sensitive. FASEB J 2005; 19: 963-5.

[68] Whitcup SM, DeBarge LR, Caspi RR, Harning R, Nussenblatt RB, Chan CC. Monoclonal antibodies against ICAM-1 (CD54) and
LFA-1 (CD11a/CD18) inhibit experimental autoimmune uveitis. Clin Immunol Immunopathol 1993; 67: 143-50.

[69] Smith JR, O'Rourke LM, Becker MD, et al. Anti-rat ICAM-1 antibody does not influence the course of experimental melanininduced uveitis. Curr Eye Res 2000; 21: 906-12.

[70] Durr E, Yu J, Krasinska KM, et al. Direct proteomic mapping of the lung microvascular endothelial cell surface in vivo and in cell culture. Nat Biotechnol 2004; 22: 985-92.

[71] Huang X, Bennett M, Thorpe PE. Anti-tumor effects and lack of side effects in mice of an immunotoxin directed against human and mouse prostate-specific membrane antigen. Prostate 2004; 61: 111.

[72] Kinnecom K, Pachter JS. Selective capture of endothelial and perivascular cells from brain microvessels using laser capture microdissection. Brain Res Brain Res Protoc 2005; 16: 1-9.

[73] Arap W, Kolonin MG, Trepel M, et al. Steps toward mapping the human vasculature by phage display. Nat Med 2002; 8: 121-7.

[74] Kolonin MG, Sun J, Do KA, et al. Synchronous selection of homing peptides for multiple tissues by in vivo phage display. FASEB J 2006; 20: 979-81.

(C) Bhattacharjee and Smith; Licensee Bentham Open.

This is an open access article licensed under the terms of the Creative Commons Attribution Non-Commercial License (http://creativecommons.org/licenses/by$\mathrm{nc} / 3.0 /$ ) which permits unrestricted, non-commercial use, distribution and reproduction in any medium, provided the work is properly cited. 